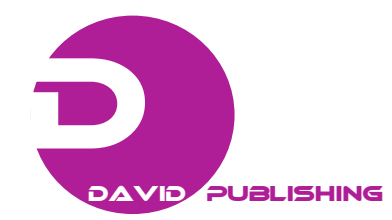

\title{
Determination of Bacterial Cell Number to Differentiate Campylobacter jejuni from C. coli by the Simple Hippurate Hydrolysis Test
}

\author{
Miyuki Fujioka* and Yoshimitsu Otomo \\ Hirosaki University Graduate School of Health Sciences, Hon-cho 66-1, Hirosaki, Aomori, 036-8564, Japan \\ *Corresponding author's e-mail: mfujioka@cc.hirosaki-u.ac.jp
}

\begin{abstract}
Campylobacter is widely known as one of the most common food borne pathogens which causes acute bacterial diarrhea in humans worldwide and C. jejuni and C. coli are the predominant bacteria that causes Campylobacteriosis. C. jejuni usually hydrolyzes hippuric acid to benzoic acid and glycine and this simple hippurate hydrolysis test is used to rapidly distinguish $C$. jejuni from $C$. coli in many clinical microbiological laboratories. However, sometimes the test gives variable results, which might be due to the inadequate number of bacterial cells taken in the test sample. In this study, the authors investigated the optimum number of bacterial cells required to perform the test smoothly. A total of $40 \mathrm{C}$. jejuni and $10 \mathrm{C}$. coli strains were included in this study and test bacterial cell suspensions were prepared matching the McFarlandunit $0.5,1$, 2, 3, 4, and 5 . Ninhydrin was added to the cell suspension and the color change was measured at an optical density of $540 \mathrm{~nm}$. Results showed that the test bacterial suspensions of C. jejunimatching McFarlandunit 1, 2, 3 , 4 and 5, were all positive. On the other hand, bacterial cell suspension of C. coli matching more than 5 gave false positive results. Reproducible results were obtained with a bacterial suspension which matched with the McFarlandunit 1 and this number of bacteria may be used for the hippurate hydrolysis test to identify C. jejuni from C. coli.
\end{abstract}

Key words: Identification, Campylobacter jejuni, hippurate hydrolysis test, Campylobacter coli. 